\title{
Short-Term Prediction for Nuclear Power Plant Failure Scenarios Using an Ensemble-based Approach
}

\section{J. Liu \& V. Vitelli}

Chair on Systems Science and the Energetic challenge, European Foundation for New Energy - Electricité de

France, École Centrale Paris, Chatenay-Malabry, France, and Supelec (École Supérieure d'Électricité),

Plateau de Moulon, Gif-sur-Yvette, France

\section{R. Seraoui}

EDF R\&D, Simulation and information TEchnologies for Power generation system (STEP) Department, Chatou, France

\author{
F. Di Maio \& E. Zio \\ Energy Department, Politecnico di Milano, Milano, Italy
}

ABSTRACT: An ensemble-based approach is proposed for the short-term prediction. The proposed approach includes the selection of the inputs using Fuzzy Similarity Analysis (FSA), Probabilistic Support Vector Regression (SVR) model as the single model of the ensemble, and the derivation of the Prediction intervals associated with the predicted value. A case study is shown, regarding the prediction of a drifting process parameter of a Nuclear Power Plant (NPP) component.

\section{INTRODUCTION}

In Nuclear Power Plant (NPP) systems, effective prediction methods are sought to anticipate, diagnose and control abnormal events in a timely manner, and to prevent high economic losses in case of unexpected faults (Venkatasubramanian, 2005). Condition monitoring of NPP components is usually performed at regular intervals, in order to effectively identify faults possibly affecting the system state. The prediction of the failure behavior evolution is also of interest for the purpose of maintenance, and for informing decisions on the actions to take to recover the system (Zio, 2012).

Considering the huge amount of data arising from NPP components condition monitoring, data-driven approaches are often the most suited for real applications in the context of complex systems. Some research work concerns data-driven approaches for condition monitoring of engineering systems. In particular, Support Vector Regression (SVR) is a learning machine implementing the Structural Risk Minimization (SRM) inductive principle to obtain good generalization performance on a limited number of learning patterns (Vapnik et al., 1996). SVR is used in Trontl et al. (2007) and Bae et al. (2008) for performing point estimation with satisfactory results in the context of multi-layer shields and nuclear reactor. In Elnokity et al. (2012), a hybrid modeling combined with the Industrial Source Complex (ISC) model and an Adaptive Neuro-Fuzzy Inference System (ANFIS) has been used to improve the modeling ability of predicting tracer concentrations. SVR method is used in Cai (2012) to predict the critical heat flux, while Fuzzy Neural Networks are used in $\mathrm{Na}$ et al. (2006) to estimate the collapse moment due to the wall-thinned defects of bends and elbows in piping systems. However, uncertainty quantification is not included in the previously described datadriven models. In Zio et al. (2010) and Zio and Di Maio (2010), a fuzzy similarity analysis is introduced to compare the evolving failure scenario with a library of reference patterns describing the multidimensional evolution of monitored process variables. The aim is to find a combination of the reference patterns, weighed by their similarity to the observed failure scenario, to determine the future evolution of the observed scenario and to derive the corresponding RUL. However, failure patterns in NPP components are rare and thus a "solid" library of references cannot be easily formed. SVR has also been used in Kim et al. (2012) to build Prediction Intervals (PIs) for the collapse moments of wallthinned pipe bends and elbows. However, since the method has been trained on a relatively small amount of data, its generalization power is not assured.

After the Bayesian probabilistic paradigm has been introduced within the SVR framework, Probabilistic Support Vector Regression (PSVR) has been defined, which can give a point estimation of the target of interest along with the associated PIs. The authors prove that the proposed modified PSVR can give better results than some other data-driven approaches in the real case study of a NPP component (Liu et al., 2013). Indeed, in Liu et al. (2013) considers only one failure scenario is considered, and the model is built only on the historical data of this scenario. In the present work, the authors try to use PSVR with multiple scenarios. An ensemble-based approach of PSVR models is proposed to exploit information carried by all the scenarios. Fuzzy Simi- 
larity Analysis (FSA) in Zio and Di Maio (2010) is applied to determine the best inputs for the PSVR model among the training data. The proposed approach gives satisfactory results in the case study of interest, concerning several NPPs of Electricité De France (EDF).

The paper is structured as follows. Section 2 provides an overview of PSVR, FSA and of the procedure that combines them. The real case study with the results is introduced in Section 3. Some conclusions are drawn in section 4.

\section{METHODOLOGY}

Suppose that $N$ recorded scenarios are available, which are representatives of the evolution concerning relevant signals of the same failure. Then the prediction of the target of the ongoing failure scenario at the next time step can be based not only on the historical values of the ongoing failure, but also on the information contained in all the $N$ recorded scenarios. These recorded scenarios are taken as references with respect to the ongoing scenario to be predicted: the former will be named "Reference Scenarios" and the latter "Observed Scenario".

Suppose that $M$ process variables are measured for each scenario, among which one is the target $b$ and the other $M-1$ are variables $\mathbf{a}=\left(\mathrm{a}_{1}, \mathrm{a}_{2}, \ldots, \mathrm{a}_{\mathrm{M}-1}\right)$ related to the target $b$.

The authors propose an ensemble-based approach to give the predicted values of the target and the associated PIs. Each model of the ensemble is built using PSVR with the Observed Scenario and one Reference Scenario. FSA is used to determine the best inputs for each PSVR model.

The inputs of the PSVR model to predict the target of the Observed Scneario $b(t+1)$ are the $M-1$ related variables of the Observed Scenario at time $t$, i.e. $\mathbf{a}(t)$, three historical values of the target: $b(t), b(t$ - 1$), b(t-2)$, and one target value $b_{\mathrm{RS}}\left(t_{0}+1\right)$ in one of the Reference Scenarios, which is determined by FSA. A motivation for the selection of the historical values can be found in Liu et al. (2013).

In the next sub-sections, the details of FSA, PSVR and ensemble are presented.

\subsection{Fuzzy similarity analysis}

Consider a fixed vector $\mathbf{v}=\left(\mathbf{a}\left(t^{*}\right), b\left(t^{*}\right), b\left(t^{*}-1\right), b\left(t^{*}\right.\right.$ - 2)) in the Observed Scenario and a time-dependent one $\mathbf{w}(\mathrm{t})=\left(\mathbf{a}_{\mathrm{RS}}(t), b_{R S}(t), b_{R S}(t-1), b_{R S}(t-2)\right)$ in a Reference Scenario; for each time $t, w(t)$ has the same data structure as $\mathbf{v}$. The purpose is to find $t_{0}$ such that the distance score $d\left(t_{0}\right)$ between $\mathbf{w}\left(t_{0}\right)$ and $\mathbf{v}$ is minimized. In the present paper, three steps are proposed to tackle this issue.

The first step consists in calculating the Euclidean distance between $\mathbf{v}$ and $\mathbf{w}(t)$ for all times $t$ : $\delta(t)=|\mathbf{v}-\mathbf{w}(t)|$

The second step is the computation of the trajectory point wise similarity and the corresponding distance score. The point wise difference between the trajectories $\mathbf{w}(t)$ and $\mathbf{v}$ expressed by Equation 1 is evaluated with reference to an "approximately zero" fuzzy set (FS) specified by a function which maps the elements of the Euclidean distance $\delta(t)$ into the corresponding similarity value $\mu(t)$ (Joentgen et al., 1999). Common functions can be used for the definition of the FS, e.g. triangular, trapezoidal, and bellshaped. In the application illustrated in this work, the following bell-shaped function is used:

$\mu(t)=\exp \left(-\left(-\ln (\alpha) / \beta^{2}\right) \delta(t)^{2}\right)$

The arbitrary parameters $\alpha$ and $\beta$ can be set by the analyst to shape the desired interpretation of similarity into the fuzzy set: the larger the value of the ratio $-\ln (\alpha) / \beta^{2}$, the narrower the fuzzy set and the stronger the definition of similarity (Zio \& Di Maio, 2010). Then the distance score $d(t)=1-\mu(t)$ between $\mathbf{v}$ and the trajectory $\mathbf{w}(t)$ is computed.

The third step is to find $t_{0}$ which minimizes $d(t)$, and to compute the corresponding distance score $d\left(t_{0}\right)$.

Finally, the value of the target $b_{\mathrm{RS}}\left(t_{0}+1\right)$ in the Reference Scenario is also used as one input in the PSVR model.

\subsection{Probabilistic Support Vector Regression}

Different Gaussian-based versions of the PSVR method can be defined, depending on the choice of the loss function. A large number of loss functions have been proposed in the literature, e.g. Quadratic Loss Function, Laplacian Loss Function, Huber's Loss Function (Chu et al., 2002). In the proposed modified PSVR approach, the authors have chosen the $\varepsilon$-insensitive Loss Function, which enables a sparse set of support vectors to be obtained (Smola and Schölkopf, 2004). The PSVR method can give an error bar associated to the predicted value.

\subsubsection{PSVR with E-Insensitive Loss Function}

Suppose we are at time $T$, and let us assume that the input data is a $n$-dimensional set of vectors $\mathbf{X}=\left\{\mathbf{x}_{1}\right.$, $\left.\mathbf{x}_{2}, \ldots, \mathbf{x}_{\mathrm{n}}\right\}$ with $\mathbf{x}_{i}=(\mathbf{a}(t), b(t), b(t-1), b(t-2)$, $\left.b_{\mathrm{RS}}\left(t_{0}(t)+1\right)\right)$ independently drawn in $\mathbf{R}^{\mathrm{M}+3}$. Suppose we also have an independent sample of target values $\mathrm{Y}=\left\{y_{1}, y_{2}, \ldots, y_{\mathrm{n}}\right\}$, with $y_{i}=b(t+1)$, where $t$ $=T-n+i-1$ and $i=1,2, \ldots, n$. In regression methods, the final aim is to find a function $f(\mathbf{x})$ : $\mathbf{R}^{\mathrm{M}+3} \rightarrow \mathbf{R}$ describing the relation between the input data and the target.

We hereafter briefly recall the PSVR approach to the estimation of $\mathrm{f}(\mathbf{x})$; further mathematical details on the derivation of the method can be found in Gao et al. (2001).

We make the following assumptions: 
1. The training data $\Gamma=\{\mathbf{X}, \mathbf{Y}\}$ is composed by independent samples, which, given $\mathbf{f}(\mathbf{X})=$ $\left(\mathrm{f}\left(\mathbf{x}_{1}\right), \mathrm{f}\left(\mathbf{x}_{2}\right), \ldots, \mathrm{f}\left(\mathbf{x}_{\mathrm{n}}\right)\right)$, are drawn from the same probability distribution.

2. The a priori probability distribution of the unknown $\mathbf{f}(\mathbf{X})$ is $\mathrm{P}[\mathbf{f}(\mathbf{X})] \propto \exp \{-1 / 2 \times$ $\left.\|\mathrm{P} f\|^{2}\right\}$, where $\|\mathrm{P} f\|^{2}$ is a positive semi-definite operator.

3. The $\varepsilon$-insensitive loss function is chosen as loss function of the PSVR method (see Gao et al., 2001).

$$
l\left(\mathbf{x}_{i}\right)= \begin{cases}|\mathbf{x}|-\varepsilon & \text { if }|\mathbf{x}|<\varepsilon \\ 0 & \text { if }|\mathbf{x}| \geq \varepsilon\end{cases}
$$

4. The covariance function is $\mathrm{K}(\mathbf{X}, \mathbf{X})$, where the element $\mathrm{K}_{i, j}$ of the $i$-th row and $\mathrm{j}$-th column is $\mathrm{K}\left(\mathbf{x}_{i}, \mathbf{x}_{j}\right)=\exp \left\{-\left|\mathbf{x}_{i}-\mathbf{x}_{j}\right|^{2} / 2 / \gamma^{2}\right\}$, with $\mathbf{x}_{i}, \mathbf{x}_{j}$ being elements of $\mathbf{X}$, and $i, j=1, \ldots, n$.

The posteriori probability of $\mathbf{f}(\mathbf{X})$ can be written as

$$
\begin{aligned}
P[\boldsymbol{f}(\boldsymbol{X}) \mid \boldsymbol{\Gamma}]= & \frac{[G(C, \varepsilon)]^{N}}{\sqrt{\operatorname{det} 2 \pi K_{\boldsymbol{X}, \boldsymbol{X}}} P[\boldsymbol{\Gamma}]} \exp \left\{-C \sum_{\boldsymbol{x}_{i} \in \boldsymbol{X}} L_{\mathcal{\varepsilon}}\left(y_{i}-\right.\right. \\
& \left.\left.f\left(\mathbf{x}_{i}\right)\right)-\frac{1}{2} \boldsymbol{f}(\boldsymbol{X})^{T} K_{\boldsymbol{X}, \boldsymbol{X}}^{-1} \boldsymbol{f}(X)\right\}
\end{aligned}
$$

where $\mathrm{G}(\mathrm{C}, \varepsilon)=\mathrm{C} / 2 /(\mathrm{C} \times \varepsilon+1)$, and $\mathrm{K}_{\mathbf{x}, \mathbf{x}}$ is a shortened notation for the covariance matrix $\mathrm{K}\left(\mathbf{x}_{i}, \mathbf{x}_{j}\right)$.

The Maximum A Posteriori (MAP) solution of Equation 4 is found by finding the minimum of the following function

$$
\begin{aligned}
& R_{G S V M}(f)= \\
& C \sum_{\mathbf{x}_{i} \in \boldsymbol{X}} L_{\varepsilon}\left(y_{i}-f\left(\mathbf{x}_{i}\right)\right)+\frac{1}{2} \boldsymbol{f}(\boldsymbol{X})^{T} K_{\boldsymbol{X}, \boldsymbol{X}}^{-1} \boldsymbol{f}(\boldsymbol{X}) .
\end{aligned}
$$

Following the discussion in Mackay (1997), we can write the solution of the minimization problem associated to Equation 5 in the following form

$f^{*}(\mathbf{x})=\sum_{\mathbf{x}_{i} \in \mathbf{X}} \beta_{i} K\left(\mathbf{x}_{i}, \mathbf{x}\right)$

where $\beta_{i}=a_{i}-a_{i}^{*}$ is a combination of the Lagrange Multipliers associated to the optimization problem (Smola and Schölkopf, 2004) and $\mathbf{x}$ is a new input vector. The $a_{i}$ and $a_{i}^{*}$ can be determined by a Quadratic Programming approach. According to Smola and Schölkopf (2004), $\forall i=1, \ldots, n, a_{i}$ and $a_{i}^{*}$ lie in the interval $[0, \mathrm{C}]$, and $\beta_{i}$ consequently lies in the interval $[-\mathrm{C}, \mathrm{C}]$, which is the domain of the optimization problem.

\subsubsection{Error Bar Estimation}

In the Bayesian treatment of the prediction problem, error bars arise naturally from the predictive distribution. They are made up of two terms, one due to the a posteriori uncertainty (the uncertainty of $f(\mathbf{x})$ ) and the other due to the intrinsic noise in the data (Kim et al., 2012). Suppose that $\mathbf{x}$ is a test input vector, and that the corresponding value of the target is the random variable $y$, obtained adding to $f(\mathbf{x})$ as unknown noise $\delta$ with zero mean; then,
$P[\boldsymbol{\Gamma} \mid \boldsymbol{f}(\boldsymbol{X})] \propto \exp \left(-C \sum_{i=1}^{n} l\left(\delta_{i}\right)\right)$

We can also obtain the density of the noise $\delta$

$P[\delta]=\frac{C}{2(C \varepsilon+1)} \exp \left(-C l_{\varepsilon}(\delta)\right)$

and the noise variance

$\sigma_{\delta}^{2}=\frac{2}{C^{2}}+\frac{\varepsilon^{2}(C \varepsilon+3)}{3(C \varepsilon+1)}$

The conditional probability distribution of $f\left(\mathbf{x}_{\mathrm{i}}\right)$ given $\Gamma$, can instead be written as

$P[f(\mathbf{x}) \mid \boldsymbol{\Gamma}]=\frac{1}{\sqrt{2 \pi} \sigma_{t}} \exp \left\{-\frac{\left(f(\boldsymbol{x})-f^{*}(\boldsymbol{x})\right)^{2}}{2 \sigma_{t}^{2}}\right\}$

with

$\sigma_{t}^{2}(\mathbf{x})=K(\mathbf{x}, \mathbf{x})-K_{\boldsymbol{X}_{M}, \mathbf{x}^{T}}^{T} K_{\boldsymbol{X}_{M}, \boldsymbol{X}_{M}}^{-1} K_{\boldsymbol{X}_{M}, \mathbf{x}}$

Consequently, the error bar width of the prediction corresponding to the test input point $\mathrm{x}$ is

$$
\begin{aligned}
\sigma^{2}(\mathbf{x})= & \sigma_{\delta}^{2}+\sigma_{t}^{2}(\mathbf{x})=\frac{2}{C^{2}}+\frac{\varepsilon^{2}(C \varepsilon+3)}{3(C \varepsilon+1)}+K(\mathbf{x}, \mathbf{x})- \\
& K_{\boldsymbol{X}_{M}, \mathbf{x}}^{T} K_{\boldsymbol{X}_{M}, \boldsymbol{X}_{M}}^{-1} K_{\boldsymbol{X}_{M}, \mathbf{x}}
\end{aligned}
$$

The conditional probability distribution and the error bar are given in Equation 10 and 12. See Gao et al. (2001) for more details on the calculations.

\subsection{Ensemble-based approach}

An ensemble-based approach is obtained by combining diverse models, to obtain superior performance with respect to the one obtained with a single model. Ensemble-based approaches attempt to take advantage of each single model, by fusing results from all the models. The strategy of combining different algorithms into an ensemble has been found attractive in a wide variety of research fields. One way for obtaining a final result from an ensemble of models is to obtain the prediction as weighted sum of the single model results.

In our approach, the single models are PSVR, built by using FSA to determine the best target value $b_{\mathrm{RS}}\left(t_{0}+1\right)$ in a Reference Scenario to be used as input together with other inputs from the Observed Scenario. There are $N$ Reference Scenarios, so $N$ PSVR models are totally built, one for each Reference Scenario.

Suppose to be at time $t$. The predicted value of the ensemble of models for the target of the Observed Scenario at time $t+1$ is the combination of the predicted values of all the models, weighted by their similarity to the evolving scenario. To calculate the weight of each model, we need to find the minimum of $d_{i}(\mathrm{t})$, which is the distance score of the $i$-th Reference Scenario. Supposing $d_{i}^{*}\left(t^{*}-1\right)$ is the minimum, the weight of the $i$-th model is calculated with Equation 13.

$w_{i}=\left(1-d_{i}^{*}\left(t^{*}-1\right)\right) \exp \left(-\frac{d_{i}^{*}}{\beta}\right)$ 
After normalization of the weights so that their sum is 1 , the final weight for each model is finally obtained and thus the predicted value of the ensemble is derived. They are separately given in Equation 14 and 15.

$w_{i}=w_{i} / \sum_{j=1}^{19} w_{j}$

$\hat{y}(t+1)=\sum_{i=1}^{19} w_{i} \hat{y}_{i}(t+1)$

where $\hat{y}(t=1)$ is the predicted value of the ensemble and $\hat{y}_{i}(t+1)$ is the predicted value of the $i$-th model.

Assume $\sigma_{i}(t+1)$ is the error bar of the prediction $\hat{y}_{i}(t+1)$ of the $i$-th model. Then, the error bar of the ensemble is calculated as Equation 14

$\sigma(\hat{y}(t+1))=\sum_{i=1}^{19} w_{i} \sigma_{i}(t+1)+\operatorname{Var}(t+1)$

where $\operatorname{Var}(t+1)$ is the variance of the predicted values of the single models, and calculated as

$$
\operatorname{Var}(t+1)=\frac{1}{N-1} \sum_{i-1}^{N}\left(\hat{y}_{i}-\mathrm{E}(\hat{y})\right),
$$

with $\mathrm{E}(\hat{y})$ being the average of all the predicted values of the single models.

The paradigm of the proposed ensemble approach is shown in Figure 1.

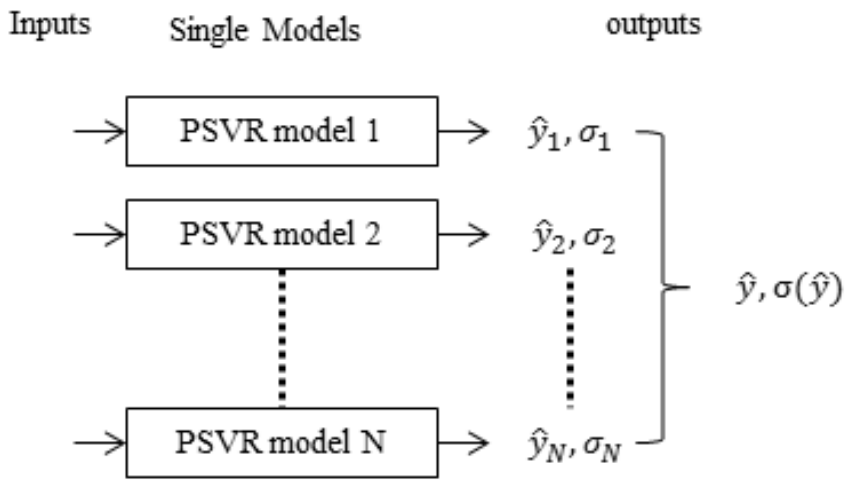

Figure 1. Paradigm of ensemble approach.

\section{CASE STUDY}

\subsection{Case study description}

Pumps play a major role in the design and operation of NPPs and of other industrial facilities. Their operating characteristics are believed to play a significant role in determining the thermal and hydraulic behavior of the nuclear reactor following transients. The Reactor Coolant Pump (RCP) is a critical component in the normal operation of a NPP, since it guarantees enough cold water in the core of the plant to protect the nuclear materials and to deliver the heat released from the nuclear fission to the heat exchanger.

In the Observed Scenario considered in this study, due to a failure the leak flow of the first seal of RCP starts drifting towards exceeding a predefined control threshold. The aim of the study is to predict the value of the leak flow in the next time point (i.e. to perform 4-hours-ahead forecasting). 19 Reference Scenarios are available from other NPPs, and they can be exploited to the prediction purposes.

All the scenarios are measured every four hours until the failure of the RCP. For each NPP, the variables are monitored starting from different time instances and for different durations; hence, the number of measurements for each NPP is different. The fault occurred at different times, and in some scenarios the operators managed to bring the pump back into a normal condition.

\subsection{Data pre-processing}

Since the dataset we are going to analyze contains both missing data and outliers, we have to deal with both these issues. Data pre-processing methods described in Liu et al (2013) are here used to eliminate the outliers and reconstruct the missing data. The data concerning the same variable of all the 20 scenarios are then normalized within the arbitrary chosen range $[0,1]$.

\subsection{Results and illustration}

200 data points are used to train each model of the ensemble and 300 to test the model. Testing results are shown in Figure 2, where the solid line is the measurements, the dashed line is the predicted value and the two dot lines are the PIs associated to a confidence level of $95 \%$, i.e. $[\hat{y}-2 \sigma(\hat{y}), \hat{y}+2 \sigma(\hat{y})]$.

The results of the prediction on the testing data are satisfactory. The coverage of the PIs is $98.33 \%$. The relative and absolute errors of the prediction are 0.0083 and 0.0032 , respectively. The average of the PIs is 0.144 .

\section{CONCLUSION}

In this paper, a short-term forecasting approach is proposed for the purposes of condition monitoring. It includes pre-processing for data reconstruction and model selection, FSA for choosing the best inputs of the PSVR model and an ensemble-based approach for estimation of the PIs of the target of interest. The results of the application to a real case study of leak flow in the first seal of a RCP are satisfactory. The coverage of the prediction interval is $98.33 \%$ with a confidence level of $95 \%$.

The future work will focus on the development of a method to compute the Remaining Useful Life (RUL) on the basis of the prediction of the target parameter of interest. This entails verifying the adaptivity of the model and updating the model when the results cannot satisfy some relevant predefined criteria. 


\section{REFERENCES}

Bae, I.H., Na, M.G., Lee, Y.J., Park, G.C. 2008. Calculation of the power peaking factor in a nuclear reactor using support vector regression models. Ann. Nucl. Energy 35: 22002205.

Cai, J.J. 2012. Applying support vector machine to predict the critical heat flux in concentric-tube open thermosiphon. Ann. Nucl. Energy 43: 114-122.

Chu W., Keerthi S. and Ong C. J. 2002. Bayesian Support Vector Regression Using a Unified Loss Function. Journal of Latex Class. Files 1 (11): 1-14

Elnokity, O., Mahmoud, I.I., Refai, M.K., Farahat, H.M. 2012. ANN based sensor faults detection isolation and reading estimates - SFDIRE: applied in a nuclear process. Ann. Nucl. Energy 49: 131-142.

Gao J.B., Gunn S.R., Harris C.J., Brown M. 2001. A Probabilistic Framework for SVM Regression and Error Bar Estimation. Mach. Learn. 46(1-3): 71-89

Kim, D.S., Kim, J.H., Gyunna, M., Kim, J.W. 2012. Uncertainty analysis of data-based models for estimating collapse moments of wall-thinned pipe bends and elbows. Nucl. Eng. Technol. 44 (3): 323-330.

Liu, J., Seraoui, R., Vitelli, V., Zio, E. 2013. Nuclear Power Plant Components Condition Monitoring by Probabilistic Support Vector Machine. Ann. Nucl. Energy in press, DOI: 10.1016/j.anucene.2013.01.005.
Na, M.G., Kim, J.W., Moreton, D.N. 2006. Estimation of collapse moment for the wall-thinned pipe bends using fuzzy model identification. Nucl. Eng. Des. 236: 1335-1343.

Smola A.J. \& Schölkopf B. 2004. A tutorial on support vector regression, Stat. \& Comp. 14(3): 199-222

Trontl, K., Smuc, T., Pevec, D. 2007. Support vector regression model for the estimation of c-ray buildup factors for multilayer shields. Ann. Nucl. Energy 34: 939-952.

Vapnik,V.N., Golowich, S.E., Smola, A. 1996. Support vector method for function approximation, regression estimation, and signal processing. In: Proceedings of the 10th Neural Information Processing Systems (NIPS) Conference, Denver, Colorado, 1996.

Venkatasubramanian, V. 2005. Prognostic and diagnostic monitoring of complex systems for product lifecycle management: challenges and opportunities. Comput. Chem. Eng. 29 (6): 1253-1263.

Zio, E. 2012. Diagnostics and prognostics of engineering systems: methods and techniques. Hershey: Engineering Science Reference.

Zio, E. \& Di Maio, F. 2010. A data-driven fuzzy approach for predicting the remaining useful life in dynamic failure scenarios of a nuclear system. Reliab. Eng. Syst. Saf. 95: 4557.

Zio, E., Di Maio, F., Stasi, M. 2010. A data-driven approach for predicting failure scenarios in nuclear systems. Ann. Nucl. Energy 37 (4): 482-491.

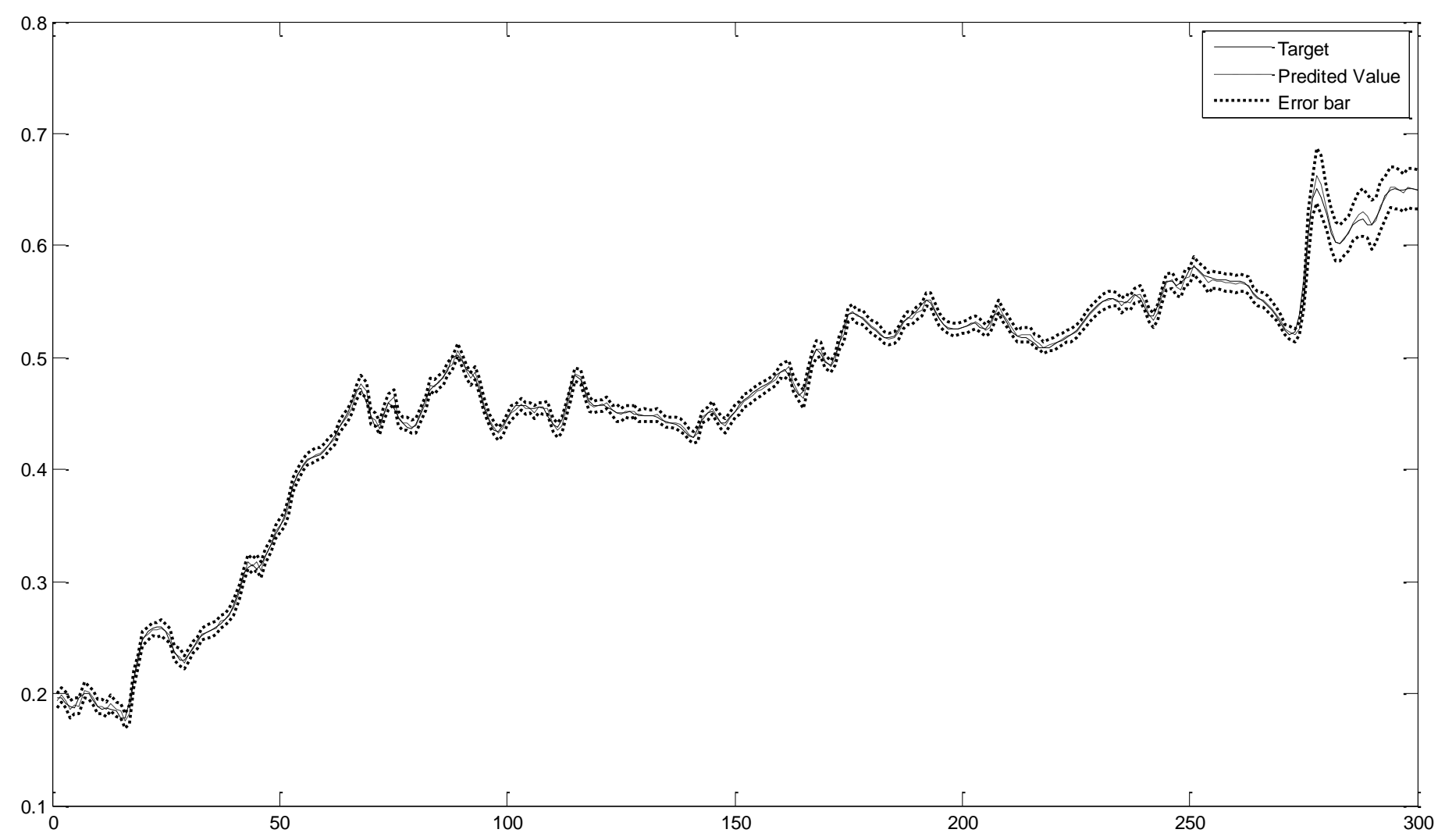

Figure 2. Point predictions and PIs of ensemble-based approach results. 